\title{
Title: A linear neural circuit for light avoidance in Drosophila larvae
}

\author{
Authors: Altar Sorkaç*, Yiannis A. Savva*†, Doruk Savaş*, Mustafa Talaył, Gilad Barnea
}

\section{Affiliations:}

Department of Neuroscience, Brown University, Providence, RI 02912, USA; Carney

Institute for Brain Science, Brown University, Providence, RI 02912, USA

$†$ Current Address: Shape Therapeutics, Inc. Seattle, WA, 98109, USA

† Current Address: Howard Hughes Medical Institute, Department of Molecular and Cellular Biology, Harvard University, Cambridge, MA, 02138, USA

* These authors contributed equally to this work

Abstract: Understanding how neural circuits underlie behaviour is challenging even in the era of the connectome because it requires a combined approach encompassing anatomical and functional analyses. This is exemplified in studying the circuit underlying the lightavoidance behaviour displayed by the larvae of the fruit fly Drosophila melanogaster. While this behaviour is robust and the nervous system relatively simple, only bits and pieces of the circuit have been delineated ${ }^{1}$. Indeed, some studies resulted in contradicting conclusions regarding the contributions of various neuronal types to this behaviour ${ }^{2,3}$. Here we devise trans-Tango MkII, a new version of the transsynaptic circuit tracing and manipulation tool trans-Tango ${ }^{4}$. We implement trans-Tango MkII in anatomical tracing and combine it with circuit epistasis analysis. We use neuronal inhibition to test necessity of particular neuronal types for light-avoidance. We complement these experiments by 

selective neuronal activation to examine sufficiency in rescuing light-avoidance deficiencies exhibited by photoreceptor mutants. Together, our studies reveal a four-order, linear circuit for light-avoidance behaviour connecting the light-detecting photoreceptors with a pair of neuroendocrine cells via two types of clock neurons. Our combined approach could be readily expanded to other larval circuits. Further, this strategy provides the framework for studying more complex nervous systems and behaviours.

Main Text: Neural circuits underlie all brain functions including processing sensory information and controlling behaviour. Understanding circuit mechanisms necessitates the use of a multipronged approach encompassing anatomical and functional analyses. The gold standard in anatomical analysis is EM reconstruction generating a connectome, and much effort has been devoted to producing connectomes of various nervous systems of organisms with increasing complexities. However, even when studying a simple behaviour in a simple organism, analysis of several layers of connected neurons is necessary, a significant challenge when using the connectome data. Further, to truly understand the flow of information in a circuit, one must use functional approaches to manipulate elements within the circuit and observe the consequences. The light-avoidance behaviour, or photophobia, exhibited by larvae of Drosophila melanogaster is an example of a robust behaviour in a relatively simple organism. Nevertheless, our knowledge about the neural circuit mediating photophobia is patchy, and at times contradictory ${ }^{2,3}$. To initiate photophobia, light is detected by $\mathrm{Rh} 5$ photoreceptors in the larval eye, the Bolwig Organ ${ }^{2,5}$. In the central brain, the prothoracicotropic hormone (PTTH)expressing neurons are essential for photophobia ${ }^{6,7}$. How these two neuronal types are connected is less clear. 
We reasoned that the information regarding light detection by Rh5 photoreceptors could be conveyed to PTTH neurons either directly through synaptic connections or indirectly via other neurons. To reveal whether Rh5 photoreceptors are presynaptic to PTTH neurons, we took advantage of trans-Tango, a transsynaptic circuit tracing, monitoring, and manipulation tool ${ }^{4,8}$.

While trans-Tango has been effectively used to reveal synaptic connections in the adult Drosophila nervous system, background noise in larvae ${ }^{4}$ limits its utility in most larval circuits. To solve this problem, we developed a new version termed trans-Tango MkII by modifying the ligand construct (Extended Data Fig. 1). Using trans-Tango MkII, we found that Rh5 photoreceptors are not presynaptic to PTTH neurons (Extended Data Fig. 2a), indicating the existence of an indirect route.

Which neurons connect the Rh5 photoreceptors to PTTH neurons? The pacemaker clock neurons in the larval visual system are attractive candidates. Expression of the genes timeless and period (per) reveals that the larval visual system comprises nine pacemaker clock neurons: four pigment dispersing factor-expressing lateral neurons (Pdf-LaNs), one Pdf-negative lateral neuron (5 ${ }^{\text {th }}$ LaN), and two pairs of dorsal neurons: DN1s and DN2s (Fig. 1a) ${ }^{9}$. Inhibition of all clock neurons via expression of the open rectifier truncated potassium channel dORK- $\Delta \mathrm{C}^{2}$ or of the inwardrectifying potassium channel Kir2.1 (Fig. 1b) results in decreased light avoidance at 1100 or 550 
Electron microscopy (EM) reconstruction of the visual system of the first instar larva reveals that $\mathrm{Rh} 5$ photoreceptors form synapses with Pdf-LaNs and the $5^{\text {th }}$-LaN ${ }^{10}$. Using trans-Tango, we observed that this also holds true in third instar larvae (Extended Data Fig. 2b). Further, PdfLaNs have been reported to directly synapse onto PTTH neurons ${ }^{6}$. We therefore sought to silence the Pdf-LaNs by expressing Kir2.1 under the control of two different Pdf-Gal4 drivers. Remarkably, these experiments revealed that Pdf-LaNs are not required for light-avoidance behaviour (Fig. 1b). Therefore, these cells are unlikely the link between Rh5 photoreceptors and PTTH neurons. Indeed, our trans-Tango experiments indicate that PTTH neurons are not postsynaptic to Pdf-LaNs (Fig. 1d).

To reveal which of the remaining clock neurons are presynaptic to PTTH neurons, we initiated trans-Tango from different subsets. We genetically accessed the $5^{\text {th }}$-LaN with two drivers from the FlyLight collection ${ }^{11}$. These drivers are expressed strongly in the $5^{\text {th }}$-LaN alongside weak and unreliable expression patterns in other neurons ${ }^{12}$. Initiating trans-Tango with either driver revealed a faint postsynaptic signal in one of the PTTH neurons, suggesting a potential albeit weak connection with the $5^{\text {th }}$-LaN (Fig. 2a and Extended Data Fig. 3) ${ }^{12}$. We next wished to examine the two pairs of dorsal neurons. However, the drivers used to access DN1s (cry) ${ }^{13}$ or DN2s $(\mathrm{Clk} 9 \mathrm{~m})^{14}$ also label Pdf-LaNs. Nevertheless, since Pdf-LaNs do not project onto the PTTH neurons, any postsynaptic signal observed in these neurons would indicate direct synaptic input from DN1s or DN2s. Indeed, initiating trans-Tango with either driver reveals that both DN1s and DN2s are presynaptic to PTTH neurons (Fig. 2b, c). 
We next sought to functionally explore the role of each subset of clock neurons in photophobia using Kir2.1. In accordance with previously published results ${ }^{3}$, we observed that silencing the $5^{\text {th }}$-LaN or DN2s leads to decreased photophobia, suggesting that these neurons are necessary for proper light avoidance. By contrast, silencing of DN1s did not affect light avoidance at 550 lux (Fig. 3a).

We reasoned that the weak direct connection between the $5^{\text {th }}$-LaN and the PTTH neurons may not be sufficient to convey the light information from Rh5 photoreceptors. Because DN2s are presynaptic to PTTH neurons and are necessary for proper light avoidance, we initiated transTango from the $5^{\text {th }}$-LaN to examine whether DN2s constitute an indirect link. We dissected the larvae at zeitgeber time (ZT) 0 when staining with antibodies against PER reveals all clock neurons ${ }^{9}$. We observed that DN2s are postsynaptic to the $5^{\text {th }}$-LaN whereas DN1s are not (Fig. 3b). We confirmed these findings at ZT12 (Extended Data Fig. 4) when PER immunoreactivity is only observed in DN2s9 . In conclusion, both our trans-Tango and neuronal silencing experiments revealed a possible anatomical pathway connecting $\mathrm{Rh} 5$ photoreceptors and the PTTH neurons comprising the $5^{\text {th }}-\mathrm{LaN}$ and DN2s. To further examine this flow of information, we performed functional rescue experiments by activating these subsets of neurons in Rh5 null mutants.

Rh5 mutant larvae are deficient in light avoidance ${ }^{2,5}$. We reasoned that if the $5^{\text {th }}$-LaN and DN2s are indeed downstream of Rh5 photoreceptors, their activation should rescue this deficiency. To test this, we expressed the light-activated cation channel CsChrimson in different clock neurons in the $\mathrm{Rh} 5$ null background. CsChrimson can be excited at red wavelengths that are mostly not 
112 visible to Drosophila ${ }^{15}$. Hence, the red light used to activate CsChrimson would not, itself, cause

113 photophobia. In the functional rescue experiments, we tested the larvae in a modified

114 photophobia assay where half of the plate was dark, and the other half was illuminated with red

115 light to activate CsChrimson. As we anticipated, larvae expressing CsChrimson in the $5^{\text {th }}$-LaN

116 avoided the red-light half of the plate, suggesting that the activation of the $5^{\text {th }}$-LaN is indeed

117 sufficient to induce aversion, and thus, to rescue the light avoidance deficiency of $\mathrm{Rh} 5$ null

118 larvae (Fig. 4a). Likewise, we expected that expression of CsChrimson in DN2s would induce

119 aversion since these neurons connect the $5^{\text {th }}$-LaN to PTTH neurons. However, to our surprise, we

120 did not observe aversion to red light when CsChrimson was expressed using our driver for DN2s

121 and Pdf-LaNs (Fig. 4b). Therefore, we decided to examine whether larvae expressing

122 CsChrimson in DN1s would avoid the red-light half of the plate as DN1s are also presynaptic to

123 PTTH neurons. We observed that these animals did not avoid the red-light part of the plate either

124 (Fig. 4c). As is the case for DN2s, our driver for DN1s also expresses in Pdf-LaNs. Hence, we

125 hypothesized that a potential phenotype caused by activation of the Pdf-LaNs might have

126 masked the effects of DN1 or DN2 activation. Indeed, activation of the Pdf-LaNs alone resulted

127 in a slight preference for the red-light half of the plate, rather than avoidance (Fig. 4d),

128 supporting our hypothesis.

To test the effects of DN2s or DN1s exclusively, we restricted the expression of CsChrimson

131 using the corresponding Gal4 drivers in conjunction with the Gal4-suppressor, Gal80, in Pdf-

132 LaNs (Extended Data Fig. 5). Selective activation of DN2s was sufficient to elicit aversion (Fig.

133 4e), effectively rescuing the light avoidance deficiency of Rh5 null larvae. These results confirm

134 the neural circuit that connects Rh5 photoreceptors to PTTH neurons through the $5^{\text {th }}$-LaN and

DN2s. 
137 Interestingly, selective DN1 activation also led to avoidance of the red-light half of the plate lux (Fig. 3a) and reduce photophobia at $750 \operatorname{lux}^{3}$. Thus, DN1 activation may cause an ectopic

141 versus bright light) is an important factor in light aversion ${ }^{16}$. Thus, it is conceivable that DN1s mediate photophobic response to dim light. To test this possibility, we silenced DN1s at 100 lux, and indeed observed that DN1s, but not other clock neurons, are necessary for photophobia at this light intensity (Extended Data Fig. 6). Thus, DN1s may be part of another circuit that mediates avoidance of dim light.

Our study revealed a circuit consisting of four orders of neurons that connect the Rh5 photoreceptors to PTTH neurons via the $5^{\text {th }}$-LaN and DN2s (Fig. 4g). While this circuit mediates the response to bright light, our observation that DN1s are necessary for photophobic response only to low light intensity indicates the existence of an additional pathway for dim light. It is

151 noteworthy that a third, independent system has been reported in which a gustatory receptor 152 mediates photophobic response to high-intensity light ${ }^{17}$. Nonetheless, our results clarify several

153 earlier studies regarding the role of Pdf-LaNs in light avoidance ${ }^{2,3,5}$. In our experiments, Pdf-

154 LaNs are dispensable for light avoidance, yet their activation is attractive. A potential explanation is that Pdf-LaNs may modulate larval photophobia via inhibition ${ }^{6,7}$, especially since adult Pdf-LaNs are glycinergic ${ }^{18}$. In addition, our results contradict a previous study reporting 
158

159

160

161

162

163

164

165

166

167

168

169

170

171

172

173

174

175

176

177

178

179

reconstitution across synaptic partners (GRASP) that is in fact not synaptic ${ }^{19}$. Thus, the proposed connection could have been the result of a non-synaptic reconstitution of GFP due to proximity.

Our analysis of the robust light avoidance response in larvae exemplifies the importance of employing a comprehensive approach combining circuit tracing together with neuronal inhibition and activation to test necessity and sufficiency. Our circuit epistasis analysis was made possible by trans-Tango MkII, a new version of trans-Tango that allows researchers to trace and manipulate neural circuits in Drosophila larvae. The combination of a robust and user-friendly genetic tool such as trans-Tango with careful functional analysis constitutes a powerful approach that can be readily expanded to studying other circuits and behaviours.

\section{References}

1 Keene, A. C. \& Sprecher, S. G. Seeing the light: photobehavior in fruit fly larvae. Trends Neurosci 35, 104-110, doi:10.1016/j.tins.2011.11.003 (2012).

2 Mazzoni, E. O., Desplan, C. \& Blau, J. Circadian pacemaker neurons transmit and modulate visual information to control a rapid behavioral response. Neuron 45, 293-300, doi:10.1016/j.neuron.2004.12.038 (2005).

3 Keene, A. C. et al. Distinct visual pathways mediate Drosophila larval light avoidance and circadian clock entrainment. $J$ Neurosci 31, 6527-6534, doi:10.1523/JNEUROSCI.6165$10.2011(2011)$

4 Talay, M. et al. Transsynaptic Mapping of Second-Order Taste Neurons in Flies by transTango. Neuron 96, 783-795 e784, doi:10.1016/j.neuron.2017.10.011 (2017). 
1805 Hassan, J., Iyengar, B., Scantlebury, N., Rodriguez Moncalvo, V. \& Campos, A. R.

181 Photic input pathways that mediate the Drosophila larval response to light and circadian

182 rhythmicity are developmentally related but functionally distinct. J Comp Neurol 481, 266-275, 183 doi:10.1002/cne.20383 (2005).

1846 Gong, Z. et al. Two pairs of neurons in the central brain control Drosophila innate light 185 preference. Science 330, 499-502, doi:10.1126/science.1195993 (2010).

1867 Zhao, W. et al. A disinhibitory mechanism biases Drosophila innate light preference. Nat

187 Commun 10, 124, doi:10.1038/s41467-018-07929-w (2019).

1888 Snell, N. J., Fisher, J. D., Hartmann, G. G., Talay, M. \& Barnea, G. Distributed

189 Representation of Taste Quality by Second-Order Gustatory Neurons in Drosophila. Preprint at:

190 https://www.biorxiv.org/content/10.1101/2020.11.10.377382v1

1919 Kaneko, M., Helfrich-Forster, C. \& Hall, J. C. Spatial and temporal expression of the

192 period and timeless genes in the developing nervous system of Drosophila: newly identified

193 pacemaker candidates and novel features of clock gene product cycling. J Neurosci 17, 6745-

$1946760(1997)$.

19510 Larderet, I. et al. Organization of the Drosophila larval visual circuit. Elife 6,

196 doi:10.7554/eLife.28387 (2017).

19711 Jenett, A. et al. A GAL4-driver line resource for Drosophila neurobiology. Cell Rep 2,

198 991-1001, doi:10.1016/j.celrep.2012.09.011 (2012).

19912 Li, H. H. et al. A GAL4 driver resource for developmental and behavioral studies on the

200 larval CNS of Drosophila. Cell Rep 8, 897-908, doi:10.1016/j.celrep.2014.06.065 (2014). 
20113 Klarsfeld, A. et al. Novel features of cryptochrome-mediated photoreception in the brain

202 circadian clock of Drosophila. J Neurosci 24, 1468-1477, doi:10.1523/JNEUROSCI.3661-

$20303.2004(2004)$.

20414 Kaneko, H. et al. Circadian rhythm of temperature preference and its neural control in

205 Drosophila. Curr Biol 22, 1851-1857, doi:10.1016/j.cub.2012.08.006 (2012).

20615 Klapoetke, N. C. et al. Independent optical excitation of distinct neural populations. Nat

207 Methods 11, 338-346, doi:10.1038/nmeth.2836 (2014).

20816 Humberg, T. H. \& Sprecher, S. G. Age- and Wavelength-Dependency of Drosophila

209 Larval Phototaxis and Behavioral Responses to Natural Lighting Conditions. Front Behav

210 Neurosci 11, 66, doi:10.3389/fnbeh.2017.00066 (2017).

21117 Xiang, Y. et al. Light-avoidance-mediating photoreceptors tile the Drosophila larval body

212 wall. Nature 468, 921-926, doi:10.1038/nature09576 (2010).

$21318 \quad$ Frenkel, L. et al. Organization of Circadian Behavior Relies on Glycinergic

214 Transmission. Cell Rep 19, 72-85, doi:10.1016/j.celrep.2017.03.034 (2017).

21519 Gordon, M. D. \& Scott, K. Motor control in a Drosophila taste circuit. Neuron 61, 373-

216 384, doi:10.1016/j.neuron.2008.12.033 (2009). 
a

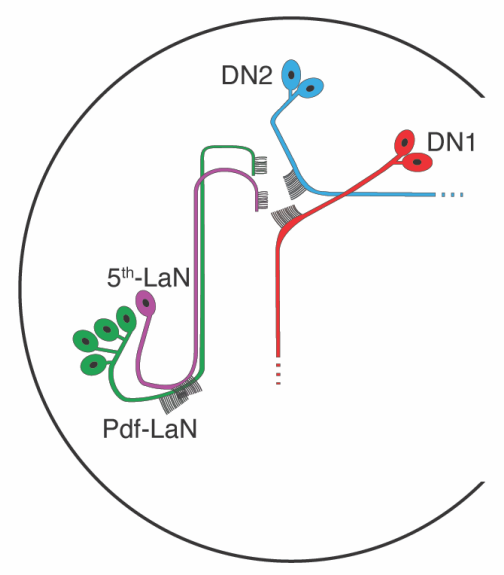

C Clock neurons $>$ trans-Tango Mkll
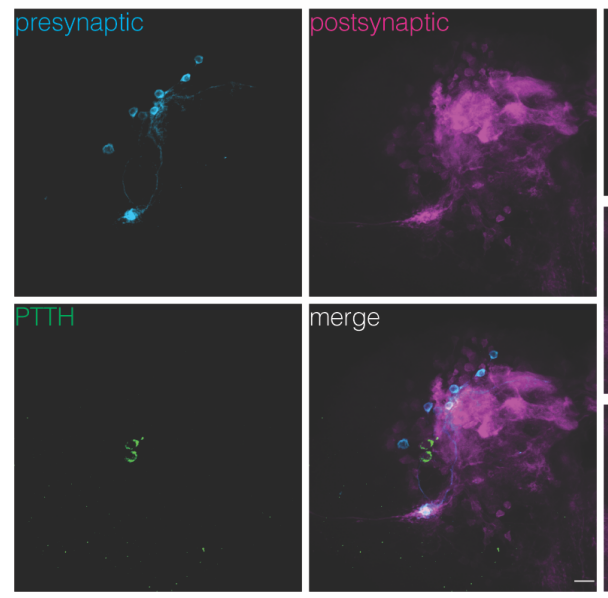

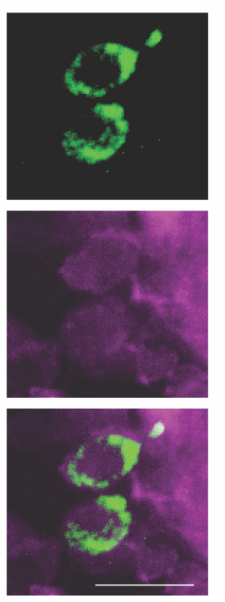

b

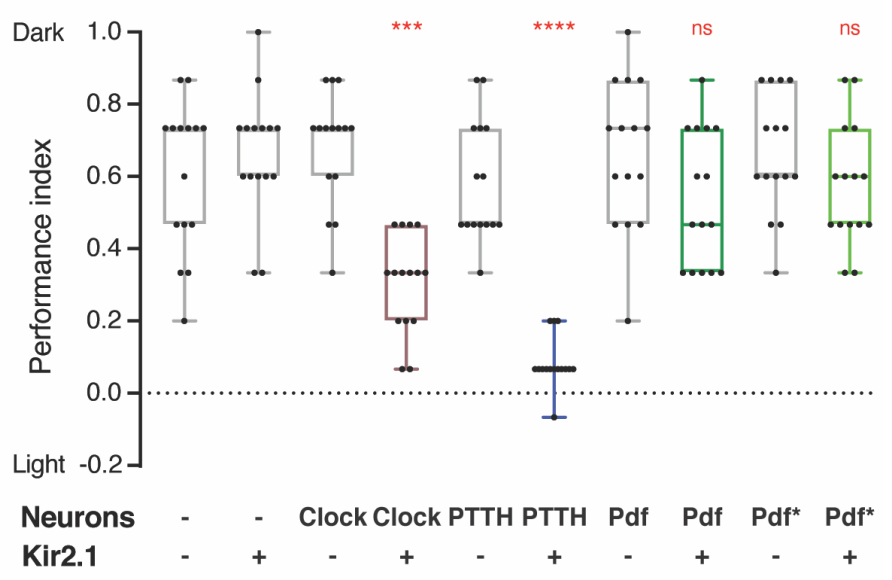

d

Pdf-LaNs > trans-Tango Mkll
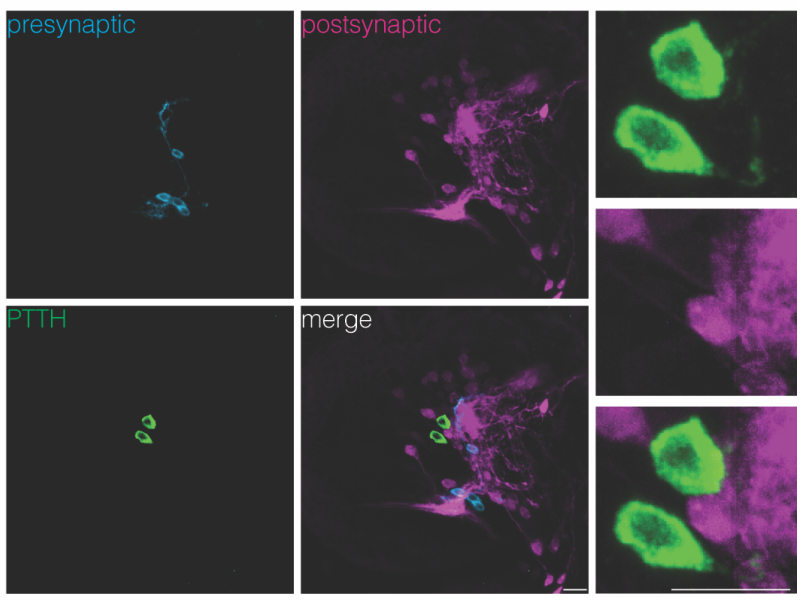

Fig. 1: Input from Pdf-negative clock neurons into PTTH neurons mediates light avoidance. a, Schematic of

clock neurons in the Drosophila larval brain. b, The effect of Kir2.1-mediated neuronal silencing on light avoidance at 550 lux. Silencing of all clock neurons or PTTH neurons decreases photophobia, silencing of Pdf-LaNs has no effect. Lines represent $75^{\text {th }}, 50^{\text {th }}$ and $25^{\text {th }}$ percentiles from top to bottom, bars represent maximum and minimum. One-way ANOVA, ns: not significant, ${ }^{* * *}$ : $\mathrm{p}<0.001, * * * *$ : $<0.0001 . \mathrm{n}=15$ trials for each group. $\mathbf{c}$, Expression of the trans-Tango MkII ligand in all clock neurons reveals postsynaptic signal in PTTH neurons. d, trans-Tango MkII reveals that PTTH neurons are not postsynaptic to Pdf-LaNs. In panels (c and d), presynaptic GFP (cyan), postsynaptic mtdTomato-HA (magenta), and PTTH (green) are shown. Scale bars, $10 \mu \mathrm{m}$. 
bioRxiv preprint doi: https://doi.org/10.1101/2021.09.09.459652; this version posted September 11, 2021. The copyright holder for this preprint (which was not certified by peer review) is the author/funder, who has granted bioRxiv a license to display the preprint in perpetuity. It is made available under aCC-BY-NC 4.0 International license.

a

$5^{\text {th }}$-LaN $>$ trans-Tango Mkll

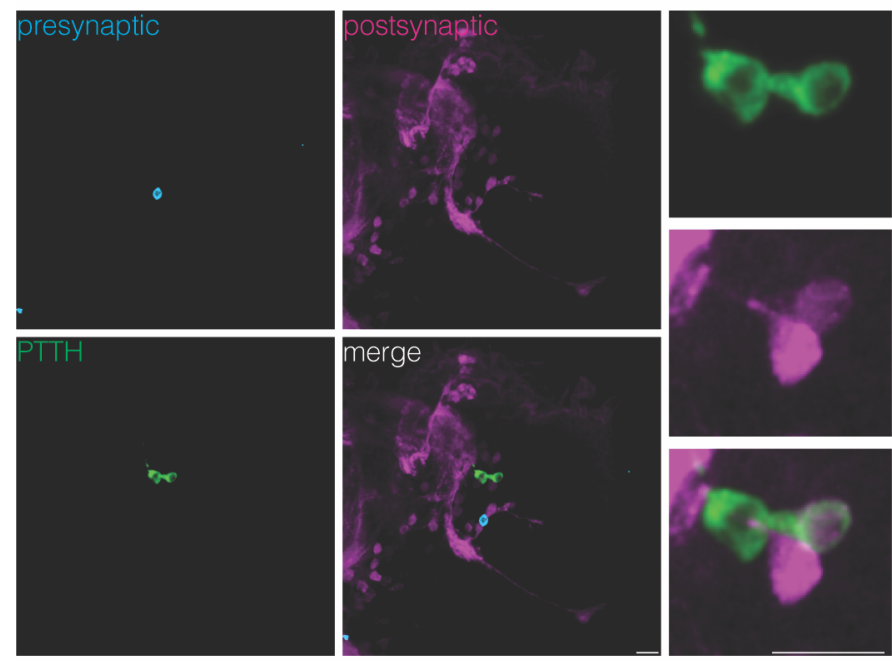

b DN1+PDF-LaNs > trans-Tango Mkll

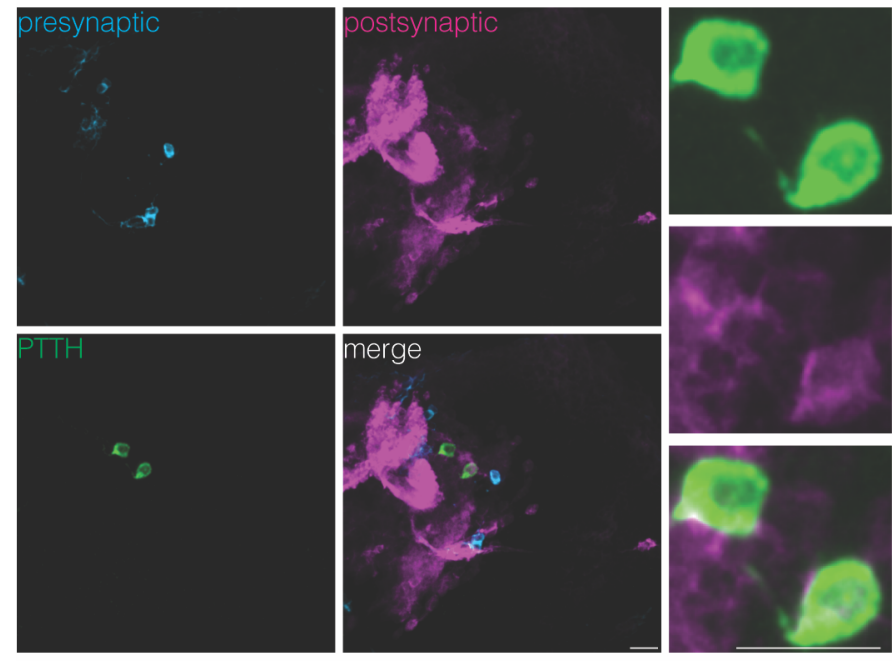

C DN2+PDF-LaNs $>$ trans-Tango Mkll

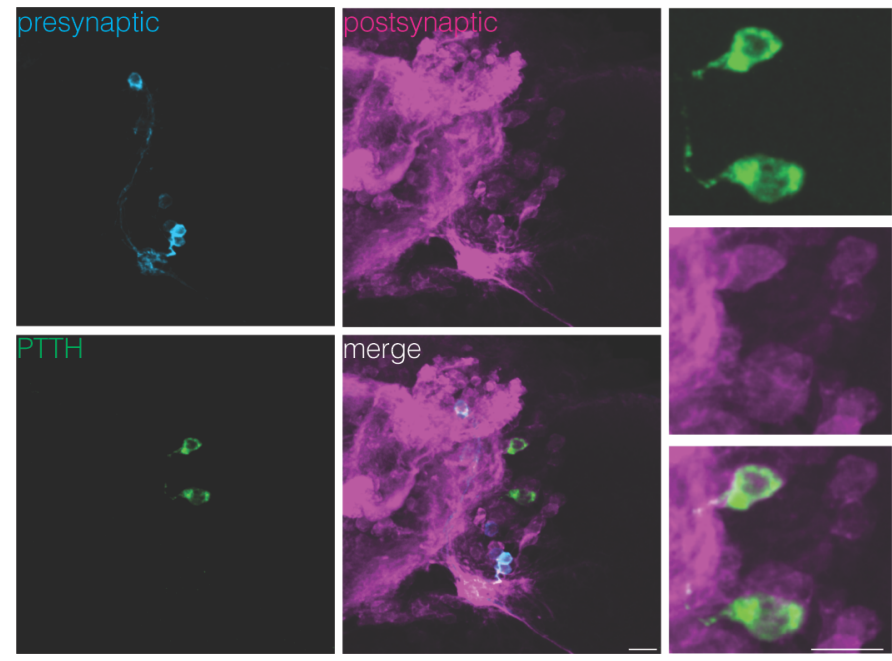


bioRxiv preprint doi: https://doi.org/10.1101/2021.09.09.459652; this version posted September 11, 2021. The copyright holder for this preprint (which was not certified by peer review) is the author/funder, who has granted bioRxiv a license to display the preprint in perpetuity. It is made available under aCC-BY-NC 4.0 International license.

236 Fig. 2: PTTH neurons receive direct input from $5^{\text {th }}$-LaN, DN1 and DN2 clock neurons. a, Only one of the

237 PTTH neurons receives input from the $5^{\text {th }}-\mathrm{LaN}$. b, c, Both PTTH neurons are postsynaptic to DN1s (b) and DN2s

238 (c). In all panels, presynaptic GFP (cyan), postsynaptic mtdTomato-HA (magenta), and PTTH (green) are shown.

239 Scale bars, $10 \mu \mathrm{m}$.

240 


\section{a}
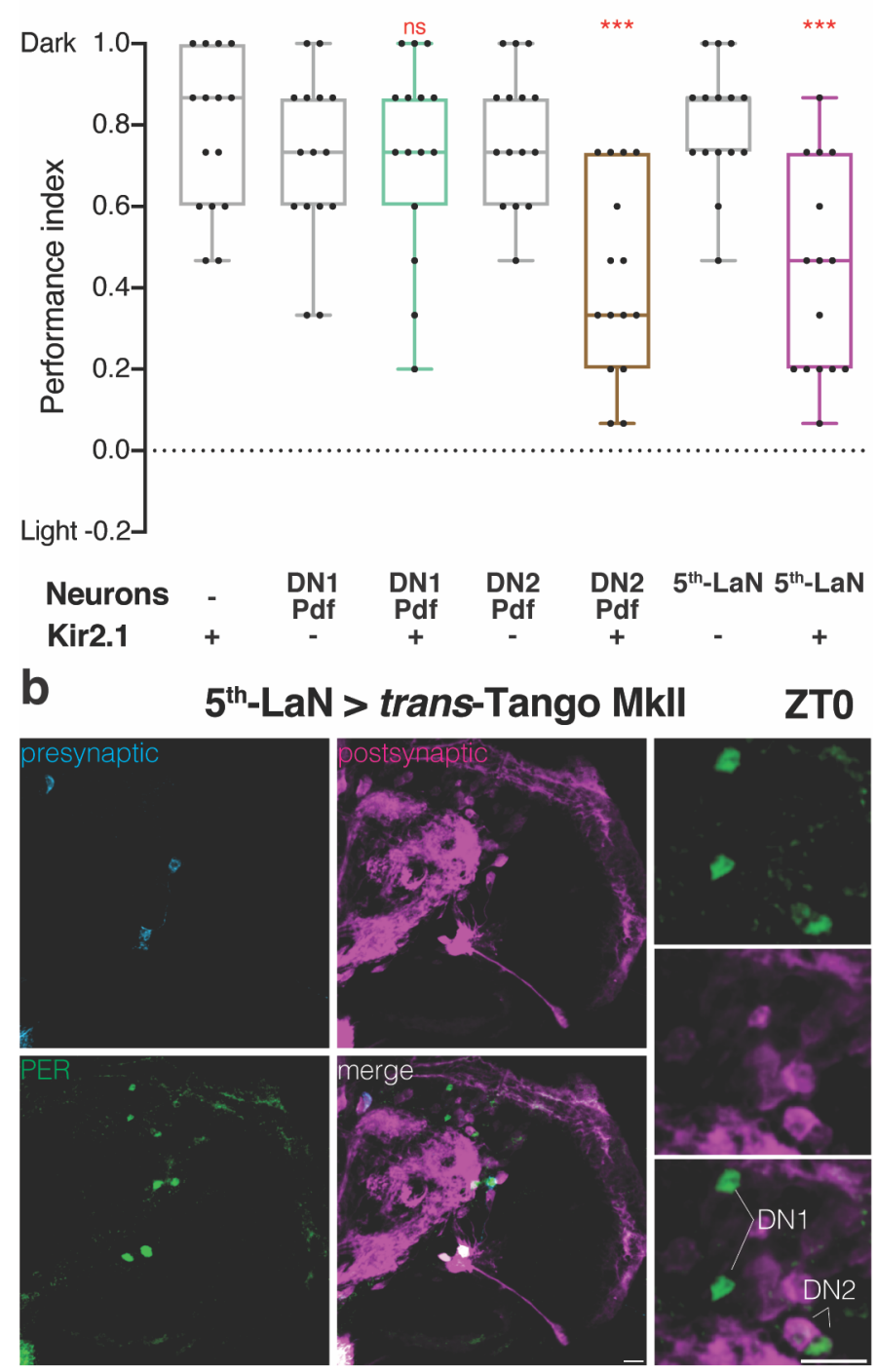

Fig. 3: Inhibition of either $5^{\text {th }}$-LaN or its postsynaptic partners DN2s reduces light avoidance. a, The effect of

243 Kir2.1-mediated silencing of clock neuron subsets on light avoidance at 550 lux. Silencing of the $5^{\text {th }}$-LaN or

244 DN2s\&Pdf-LaNs results in defective photophobia, whereas silencing of DN1s\&Pdf-LaNs has no effect. Lines

245 represent $75^{\text {th }}, 50^{\text {th }}$ and $25^{\text {th }}$ percentiles from top to bottom, bars represent maximum and minimum. One-way

246 ANOVA, ns: not significant, ***: $\mathrm{p}<0.001 . \mathrm{n}=15$ trials for each group. $\mathbf{b}, \mathrm{DN} 2 \mathrm{~s}$ but not DN1s receive direct synaptic

247 input from the $5^{\text {th }}$-LaN as revealed by PER staining in ZT0. Presynaptic GFP (cyan), postsynaptic mtdTomato-HA

248 (magenta), and PER (green). Scale bars, $10 \mu \mathrm{m}$. 


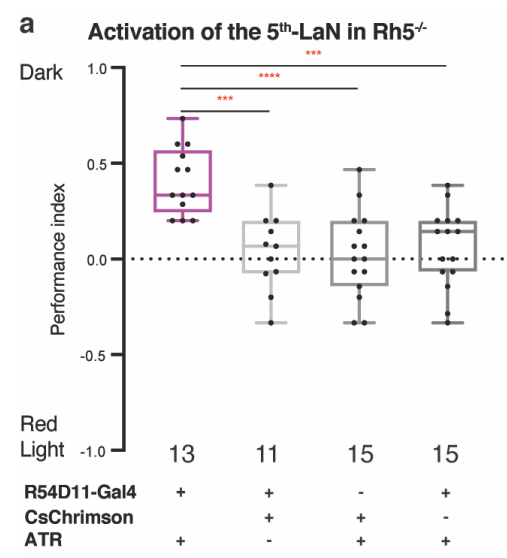

d Activation of PDF-LaNs in Rh5 ${ }^{-*}$

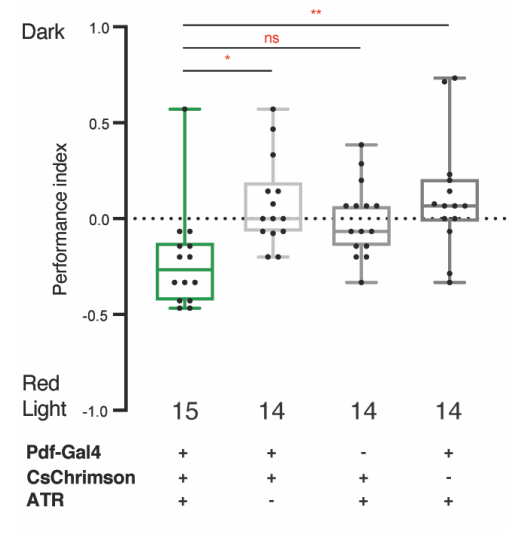

g

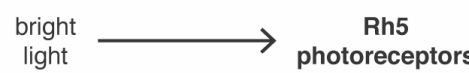
light
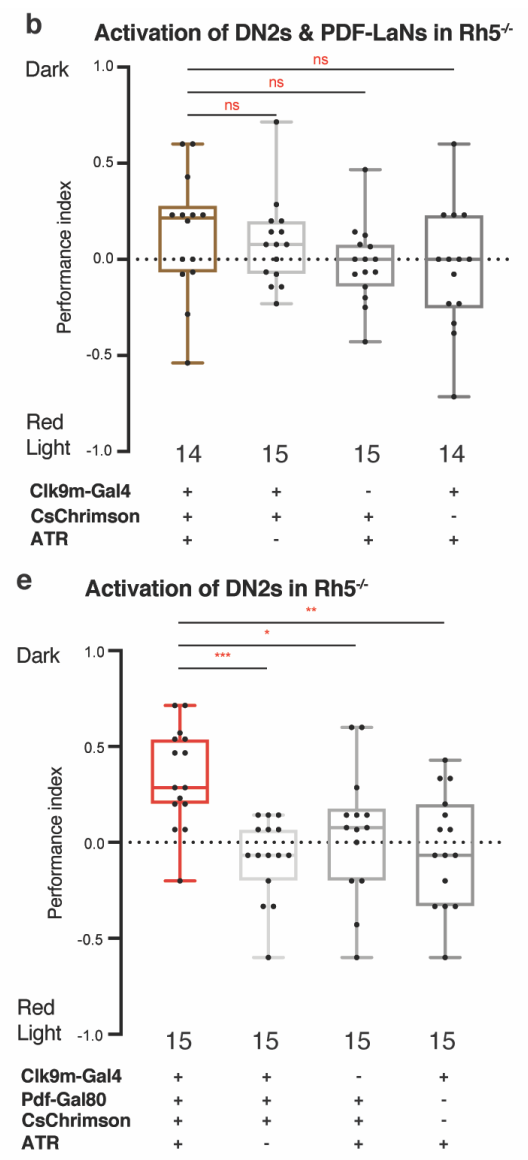
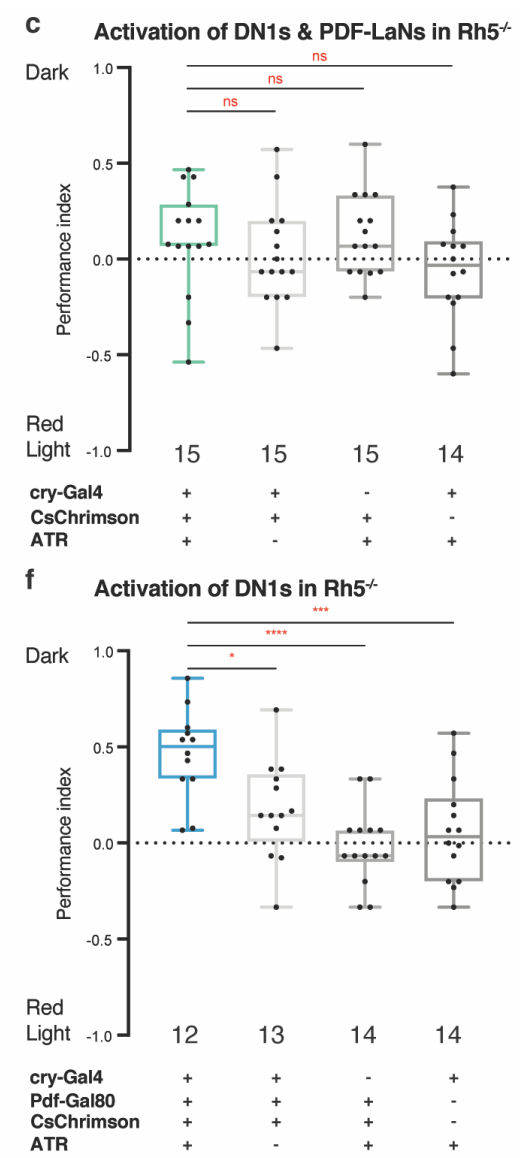
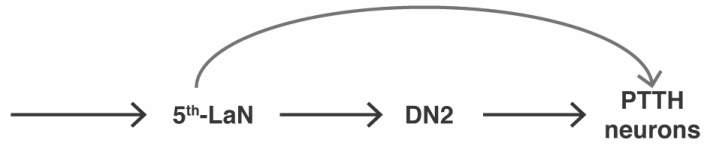

light avoidance

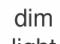

light

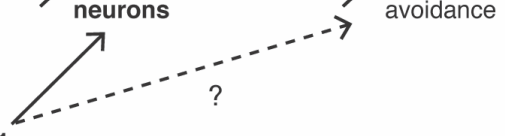

\section{Fig. 4: Activation of the $5^{\text {th }}$-LaN, DN2s or DN1s rescues the light avoidance defect exhibited by Rh5 mutant}

larvae. a-f Rescue of the light avoidance defect of Rh5 mutant larvae via CsChrimson mediated activation of specific subsets of clock neurons. Activation of the $5^{\text {th }}$-LaN (a), DN2s (e) or DN1s (f) results in light avoidance. Activation of Pdf-LaNs results in light preference (d). No effect is observed when Pdf-LaNs are activated alongside DN2s (b) or DN1s (c). ATR: all-trans retinal. Lines represent $75^{\text {th }}, 50^{\text {th }}$ and $25^{\text {th }}$ percentiles from top to bottom, bars represent maximum and minimum. One-way ANOVA, ns: not significant, *: $<<0.05,{ }^{* *}: \mathrm{p}<0.01,{ }^{* * *}: \mathrm{p}<0.001$, ****: $\mathrm{p}<0.0001$. The number of trials for each group is indicated below each box. $\mathbf{g}$, A schematic showing the flow of information in the neural circuit that mediates the light avoidance behaviour. Bright light activates Rh5 photoreceptors that convey this information to PTTH-neurons via the $5^{\text {th }}$-LaN and DN2s to mediate light avoidance.

Dim light indirectly activates DN1s that convey this information to PTTH neurons to mediate light avoidance. 
Methods:

\section{Fly Strains}

264 All fly lines used in this study were maintained at $25^{\circ} \mathrm{C}$ on standard cornmeal-agar-molasses

265 media in humidity-controlled incubators under $12 \mathrm{~h}$ light/dark cycle, unless otherwise stated. Fly

266 lines used in this study are in Table 1.

\begin{tabular}{|c|c|c|c|}
\hline Drosophila Strains & Expression pattern & Associated Figures & Stock \\
\hline Clk856-Gal423 & All Clock neurons & $1 \mathrm{~B}, 1 \mathrm{C}$ & \\
\hline NP0394-Gal4 & PTTH neurons & $1 \mathrm{~B}$ & DGRC_K_103604 \\
\hline Pdf-Gal4 & Pdf-LaNs (as Pdf) & $1 \mathrm{~B}, 1 \mathrm{D}, 4 \mathrm{D}$ & $\begin{array}{l}\text { Isolated from } \\
\qquad \text { BDSC_25031 }\end{array}$ \\
\hline DvPdf-Gal4 ${ }^{24}$ & Pdf-LaNs (as Pdf*) & $1 \mathrm{~B}$ & \\
\hline R54D11-Gal4 ${ }^{11}$ & $\begin{array}{l}5^{\text {th }} \text {-LaN, weak and } \\
\text { unreliable } \\
\text { expression in other } \\
\text { neurons }\end{array}$ & $2 \mathrm{~A}, 3 \mathrm{~A}, 3 \mathrm{~B}, 4 \mathrm{~A} \mathrm{~S} 4, \mathrm{~S} 6$ & BDSC_41279 \\
\hline cry-Gal4 ${ }^{13}$ & DN1s and Pdf-LaNs & $\begin{array}{l}2 \mathrm{~B}, 3 \mathrm{~A}, 4 \mathrm{C}, 4 \mathrm{~F}, \mathrm{~S} 5 \mathrm{~A} \\
\quad \mathrm{~S} 6\end{array}$ & \\
\hline $\mathrm{Clk} \mathrm{m}-\mathrm{Ga} 14^{9}$ & DN2s and Pdf-LaNs & $\begin{array}{l}2 \mathrm{C}, 3 \mathrm{~A}, 4 \mathrm{~B}, 4 \mathrm{E}, \mathrm{S} 5 \mathrm{~B} \\
\quad \mathrm{~S} 6\end{array}$ & BDSC_41810 \\
\hline
\end{tabular}


bioRxiv preprint doi: https://doi.org/10.1101/2021.09.09.459652; this version posted September 11, 2021. The copyright holder for this preprint (which was not certified by peer review) is the author/funder, who has granted bioRxiv a license to display the preprint in perpetuity. It is made available under aCC-BY-NC 4.0 International license.

\begin{tabular}{|c|c|c|c|}
\hline Rh5-Gal4 & Rh5 photoreceptors & $\mathrm{S} 2 \mathrm{~A}, \mathrm{~S} 2 \mathrm{~B}$ & BDSC_7458 \\
\hline R19C05-Gal4 ${ }^{11}$ & $5^{\text {th }}-\mathrm{LaN}$, others & $\mathrm{S} 3$ & BDSC_48842 \\
\hline Or42a-Gal4 ${ }^{25}$ & Or42a ORNs & $\mathrm{S} 1 \mathrm{~A}, \mathrm{~S} 1 \mathrm{C}$ & BDSC_9960 \\
\hline Pdf-Gal80²6 & $\mathrm{N} / \mathrm{A}$ & $4 \mathrm{E}, 4 \mathrm{~F}, \mathrm{~S} 5 \mathrm{~A}, \mathrm{~S} 5 \mathrm{~B}$ & $\begin{array}{l}\text { Isolated from } \\
\qquad \text { BDSC_80940 }\end{array}$ \\
\hline $\mathrm{w}^{1118}(5905)$ & $\mathrm{N} / \mathrm{A}$ & $1 \mathrm{~B}$ & BDSC_5905 \\
\hline $\operatorname{Rh} 5[2]^{27}$ & $\mathrm{~N} / \mathrm{A}$ & $\begin{array}{c}4 \mathrm{~A}, 4 \mathrm{~B}, 4 \mathrm{C}, 4 \mathrm{D}, 4 \mathrm{E} \\
4 \mathrm{~F}, \mathrm{~S} 5 \mathrm{~A}, \mathrm{~S} 5 \mathrm{~B}\end{array}$ & \\
\hline UAS-Kir2. $1^{28}$ & $\mathrm{~N} / \mathrm{A}$ & 1B, 3A, S6 & BDSC_6595 \\
\hline $\begin{array}{l}\text { UAS-Cshrimson- } \\
\text { mVenus }\end{array}$ & $\mathrm{N} / \mathrm{A}$ & $\begin{array}{c}4 \mathrm{~A}, 4 \mathrm{~B}, 4 \mathrm{C}, 4 \mathrm{D}, 4 \mathrm{E} \\
4 \mathrm{~F}, \mathrm{~S} 5 \mathrm{~A}, \mathrm{~S} 5 \mathrm{~B}\end{array}$ & BDSC_55136 \\
\hline $\begin{array}{l}\text { UAS-myrGFP, } \\
\text { QUAS- } \\
\text { mtdTomato-HA } 29\end{array}$ & $\mathrm{~N} / \mathrm{A}$ & $\begin{array}{c}1 \mathrm{C}, 1 \mathrm{D}, 2 \mathrm{~A}, 2 \mathrm{~B}, 2 \mathrm{C} \\
3 \mathrm{~B}, \mathrm{~S} 1 \mathrm{~A}, \mathrm{~S} 1 \mathrm{C} \\
\mathrm{S} 2 \mathrm{~A}, \mathrm{~S} 2 \mathrm{~B}, \mathrm{~S} 3, \mathrm{~S} 4\end{array}$ & BDSC_30118 \\
\hline trans-Tango ${ }^{4}$ & $\mathrm{~N} / \mathrm{A}$ & $\mathrm{S} 1 \mathrm{~A}$ & BDSC_77123 \\
\hline trans-Tango MkII & $\mathrm{N} / \mathrm{A}$ & $\begin{array}{c}1 \mathrm{C}, 1 \mathrm{D}, 2 \mathrm{~A}, 2 \mathrm{~B}, 2 \mathrm{C} \\
3 \mathrm{~B}, \mathrm{~S} 1 \mathrm{C}, \mathrm{S} 2 \mathrm{~A} \\
\mathrm{~S} 2 \mathrm{~B}, \mathrm{~S} 3, \mathrm{~S} 4\end{array}$ & This study \\
\hline
\end{tabular}

Table 1: Fly lines used in this study 
271 The plasmid trans-Tango MkII was generated using HiFi DNA Assembly (New England Biolabs

272 \#2621) and was incorporated into the attP40 locus using the ФC31 system as described in the

273 original trans-Tango paper ${ }^{4}$. Briefly the hICAM1::dNrxn1 sequences in the trans-Tango plasmid

274 were replaced by dNrxn1 sequence amplified from the cDNA clone LP14275 (DGRC \#1064347)

275 using the following primers: 5'-

276 atggtaacgggaatactagtCTAGATGGATCGCAAAACTCCTTCTAC-3' and 5'-

277 ttgttattttaaaaacgattcatggcgegccTTACACATACCACTCCTTGACGTC-3'. The resulting PCR

278 product was subsequently cloned via HiFi Assembly into the trans-Tango plasmid.

\section{Immunohistochemistry, Imaging, and Image processing}

281 Larval dissections, immunohistochemical experiments, and imaging were performed as

282 described in the original trans-Tango paper ${ }^{4}$. Unless otherwise stated, foraging third-instar larvae from vials reared at $25^{\circ} \mathrm{C}$ were dissected. The antibodies used in this study are: anti-PDF rabbit ${ }^{20}$ (a gift from Heinrich Dircksen, 1:3000), anti-PTTH guinea pig ${ }^{21}$ (a gift from Michael

O’Connor, 1:400), anti-PER mouse ${ }^{22}$ (a gift from James Jepson, 1:50000), anti-GFP rabbit (Thermo Fisher Scientific, A11122; 1:1,000), anti-HA rat (Roche, 11867423001; 1:100), antiBrp mouse (nc82; DSHB; 1:50), donkey anti-rabbit Alexa Fluor 488 (1:1000), goat anti-rat Alexa Fluor 555 (1:1000), donkey anti-mouse Alexa Fluor 647 (1:1000). Since the trans-Tango signal was too weak with R54D11-Gal4 at $25^{\circ} \mathrm{C}$, those crosses were set at $18^{\circ} \mathrm{C}$ for optimization (Fig. 2A, 3B and S4). Resultant images from trans-Tango experiments were processed using the Zen software (Zeiss) setting white, black and light corrections in all channels to provide better contrast. In trans-Tango figures zoomed-out images represent the maximum projection of the Z- 
stacks throughout the brains whereas the zoomed-in images were formed using subsets of the Zstacks for clarity.

\section{Light Avoidance Behavioural Assay}

Larval photophobic behaviour was tested as described previously ${ }^{2}$ with minor modifications. All animals used in this assay have been $6 \mathrm{X}$ backcrossed to BDSC_5905. Briefly, foraging early third instar larvae were collected from the food, washed with phosphate buffered saline (PBS) twice and let dry on a surface for 3 minutes. 13 to 16 animals were then transferred along the midline between dark and light halves of a $10 \mathrm{~cm}$ round petri dish with $15 \mathrm{~mL} 1.5 \%$ agar solution. Half of the lid was covered with a black tape to form the dark half. The plates were exposed to 100 or 550 lux of white light from above and experiments were run for ten minutes at $25^{\circ} \mathrm{C}$. At the end of the ten minutes, larvae on either half of the plate were counted and the preference index was calculated as (\# of larvae in the dark)-(\# of larvae in the light)/(total \# of larvae). For each genotype/condition, at least twelve trials were run over a three-day period. Analysis and determination of significance was performed using One-way ANOVA and subjected to Tukey's multiple comparison test. Experimental groups were compared to all control groups to determine significance, the lowest pairwise significance is indicated on the figures.

\section{Optogenetic Rescue Experiments}

Optogenetic rescue experiments were run in a similar manner to light avoidance assays with necessary modifications to accommodate for optogenetics. Instead of white light, the light half of the plates were exposed to LED red-light with an intensity of 1600 lux. In addition, parental 
crosses to obtain experimental animals were set up on standard medium supplemented with 400 $\mu \mathrm{M}$ all-trans-retinal (ATR, Sigma \#R2500) food or on a standard medium mixed with 100\% ethanol for no ATR controls. All animals were kept in $24 \mathrm{~h}$ dark.

Data and materials availability: All data are available in the main text or the supplementary materials. Raw data are available upon request. All new fly strains will be deposited to Bloomington Drosophila Stock Center.

\section{Methods References:}

20 Dircksen, H. et al. The ultrastructure of nerve endings containing pigment-dispersing hormone $(\mathrm{PDH})$ in crustacean sinus glands: Identification by an antiserum against a synthetic PDH. Cell and Tissue Research 250, 377-387, doi:10.1007/BF00219082 (1987).

21 Yamanaka, N. et al. Neuroendocrine control of Drosophila larval light preference.

Science 341, 1113-1116, doi:10.1126/science.1241210 (2013).

22 Lamaze, A., Kratschmer, P., Chen, K. F., Lowe, S. \& Jepson, J. E. C. A Wake-Promoting Circadian Output Circuit in Drosophila. Curr Biol 28, 3098-3105 e3093, doi:10.1016/j.cub.2018.07.024 (2018).

23 Gummadova, J. O., Coutts, G. A. \& Glossop, N. R. Analysis of the Drosophila Clock promoter reveals heterogeneity in expression between subgroups of central oscillator cells and identifies a novel enhancer region. J Biol Rhythms 24, 353-367, doi:10.1177/0748730409343890 (2009). 
33724 Bahn, J. H., Lee, G. \& Park, J. H. Comparative analysis of Pdf-mediated circadian

338 behaviors between Drosophila melanogaster and D. virilis. Genetics 181, 965-975,

339 doi:10.1534/genetics.108.099069 (2009).

34025 Fishilevich, E. \& Vosshall, L. B. Genetic and functional subdivision of the Drosophila

341 antennal lobe. Curr Biol 15, 1548-1553, doi:10.1016/j.cub.2005.07.066 (2005).

34226 Stoleru, D., Peng, Y., Nawathean, P. \& Rosbash, M. A resetting signal between

343 Drosophila pacemakers synchronizes morning and evening activity. Nature 438, 238-242,

344 doi:10.1038/nature04192 (2005).

34527 Yamaguchi, S., Wolf, R., Desplan, C. \& Heisenberg, M. Motion vision is independent of

346 color in Drosophila. Proc Natl Acad Sci U S A 105, 4910-4915, doi:10.1073/pnas.0711484105

347 (2008).

34828 Hardie, R. C. et al. Calcium influx via TRP channels is required to maintain PIP2 levels

349 in Drosophila photoreceptors. Neuron 30, 149-159, doi:10.1016/s0896-6273(01)00269-0 (2001).

35029 Potter, C. J., Tasic, B., Russler, E. V., Liang, L. \& Luo, L. The Q system: a repressible

351 binary system for transgene expression, lineage tracing, and mosaic analysis. Cell 141, 536-548,

352 doi:10.1016/j.cell.2010.02.025 (2010).

Acknowledgments: We would like to thank Alex Fleischmann, Stavros Lomvardas and members of Barnea Lab for critical reading of the manuscript. We are grateful to Heinrich by National Institutes of Health grants R01MH105368, R21DC014333 R01DC017146 (GB) and

358 Brown University Carney Institute for Brain Science, Suna Kıraç Fund for Brain Science (DS). 


\section{Author contributions:}

361 A.S., Y.A.S., D.S. and G.B. conceptualized the story. A.S., Y.A.S., D.S., M.T. and G.B. devised

362 the methodology. A.S., Y.A.S. and D.S. contributed to the investigation and visualization of the

363 results. A.S, Y.A.S., D.S. and G.B. administered the project. A.S., Y.A.S., D.S., M.T. and G.B.

364 wrote the manuscript. The funding was obtained by, and the project was supervised by G.B.

366 Competing interests: Authors declare that they have no competing interests.

368 Additional information: All new fly strains will be deposited to Bloomington Drosophila Stock

369 Center. Correspondence and requests for materials should be addressed to G.B. Reprints and

370 permissions information is available at www.nature.com/reprints 